\title{
Accessi venosi difficili e consumatori di sostanze: indagine sulle sedi di prelievo e sulle difficoltà di esecuzione
}

\author{
J. Acquaro, M. Portesan, E. Bignamini*
}

\section{SUMMARY}

- Introduction: injection drug users (IDUs) are a patients considered like a hard venous access category. The Addictions Department (Ser.D) screen and monitoring infectious diseases related to addiction through blood samples.

Aim: identify the mostly used site for taking of blood samples and assess whether there is difficulty in the execution.

Method: descriptive-observational study; it uses a monitoring template already used and isolates IDUs from non-injection drug users. Data is processed using Microsoft Excel ${ }^{\circledR}$. The research was carried out from 25/02/2015 to 30/09/2015 on 163 patients. In 62\% of cases blood sampling was performed in median cubital vein, respectively, $54 \%$ and $83 \%$ in IDUs in NIDU. The mean of attempts in IDU patients is 2 , in NIDU is 1. In $18 \%$ of cases were ultrasound driven. In $2 \%$ arterial puncture was required. In $2 \%$ of IDUs blood sampling was failed.

Discussion: the cubital vein is the mainly used and the first choice. In some cases, the patient requires blood withdrawal in different veins because cubital veins is used for substances or considered obliterated; in these situations the relationship is crucial. Health professionals find problems on the technical side, especially in IDUs, in finding a suitable venous access and on the relational building, balancing user requirements with the need to take the sample without altering the professional/ patient relationship. -

Keywords: Injection drug users, blood sample, nursing.

Parole chiave: Uso di sostanze per via iniettiva, prelievo ematico, assistenza infermieristica.

\section{Introduzione}

Il consumo illecito di oppiacei è responsabile di un'alta percentuale di mortalità e di morbilità dovuta al consumo di droga in Europa, dove il principale oppiaceo consumato è l'eroina.

La prevalenza media annuale del consumo ad alto rischio di oppiacei tra gli adulti (15-64 anni) nel 2013 è stata stimata all'incirca allo $0,4 \%$, equivalente a 1,3 milioni di consumatori problematici in Europa.

I consumatori di oppiacei (soprattutto di eroina) come droga primaria rappresentano il $41 \%$ di tutti i consumatori di stupefacenti che nel 2013 si sono sottoposti a trattamento specialistico per la tossicodipendenza (175.000 pazienti) e il 20\% di quelli che si sono sottoposti a trattamento per la prima volta (31.000 pazienti). I consumatori di sostanze stupefacenti per via parenterale (injection drug users - IDU) figurano fra i soggetti che corrono il rischio più elevato di andare incontro a problemi di salute, in particolare infezioni trasmissibili per via ematica e overdose da stupefacenti (1).

La maggior parte dei consumatori inizia la pratica di iniezione a partire da piccole vene periferiche dell'avambraccio o dalla fossa cubitale.

* Dipartimento di Patologia delle Dipendenze 1, ASL TO 2.
Poiché queste vene diventano più difficili da utilizzare, i consumatori passano gradualmente ad altri siti (2-3): la mano, il piede, la gamba, il collo e l'inguine (4-5).

Per questi motivi, la popolazione dipendente da sostanze d'abuso per via iniettiva è una delle categorie di pazienti in cui l'accesso venoso è considerato difficile (6-7).

I Servizi per le Dipendenze, rispondendo al mandato istituzionale di sanità pubblica, effettuano attività di screening e monitoraggio delle malattie infettive correlate all'uso di sostanze.

Il prelievo venoso è lo strumento diagnostico di elezione per il monitoraggio di questi pazienti (8).

La presente ricerca ha lo scopo di individuare la sede maggiormente utilizzata per il prelievo ematico e valutare se vi sia un'effettiva difficoltà nell'esecuzione.

\section{Metodo}

Lo studio è stato eseguito presso il Dipartimento Dipendenze 1 dell'Asl TO2.

Il luogo in cui si è svolta la ricerca è l'ambulatorio di Pronta Assistenza (PR.ASSI), un Servizio a bassa soglia che offre una rapida valutazione dei bisogni degli utenti tossicodipendenti ed un rapido primo trattamento e/o eventuale invio o accompagnamento 
ad altra struttura sul territorio (DEA, ambulatori specialistici, MMG).

L'ambulatorio, nella giornata di mercoledì, dedica una stanza per 3,5 ore all'effettuazione di prelievi ematici di routine e screening infettivologici per i pazienti in carico ai vari ambulatori del Dipartimento.

Il personale in turno è composto da medici e infermieri, per un totale di almeno tre operatori (due infermieri e un medico o due medici e un infermiere).

I prelievi vengono effettuati prevalentemente dagli infermieri, che si avvalgono di un ecografo in caso di difficoltà già nota nell'esecuzione del prelievo o in altri casi, dopo alcuni tentativi senza I'ausilio di questo strumento.

Lo studio, di tipo descrittivo osservazionale, è stato effettuato analizzando i dati della griglia di registrazione comunemente usata all'interno del Servizio.

La griglia, costruita ad hoc, è costituita da sette items: dati anagrafici del paziente, ambulatorio di appartenenza, medico di riferimento, tipo di prelievo effettuato (routine, virologico di primo o secondo livello), sede del prelievo, numero di tentativi, utilizzo dell'ecografo.

Ai fini della ricerca vengono presi in considerazione:

- sede del prelievo;

- numero di tentativi;

- eventuale impiego dell'ecografo.

Si è presa inoltre in considerazione la modalità di utilizzo della sostanza, rilevabile dalla cartella clinica, al fine di poter distinguere la popolazione IDU da quella dei consumatori che utilizzano altre modalità di assunzione (non injection drug users NIDU).

Il periodo di ricerca è stato scelto sulla base dell'entrata a regime dell'utilizzo dell'ecografo, elemento da poco introdotto all'interno del Servizio.

I dati sono stati elaborati attraverso il software Microsoft Excel@.

\section{Risultati}

Il periodo di osservazione è compreso tra il 25/02/2015 e il 30/09/2015

Sono state svolte 29 sedute per un totale 178 prelievi (media di 6 prelievi per seduta).

Il campione di 163 pazienti è composto da 125 IDU e 53 NIDU. L'accesso più utilizzato per il prelievo dei campioni è stata la vena mediana cubitale in entrambe le categorie di pazienti rispettivamente nel $54 \%$ degli IDU e nell' $83 \%$ dei NIDU. Nella maggioranza dei casi il prelievo è stato effettuato con un solo tentativo, mentre nel $26 \%$ sono stati necessari due o più tentativi.

Più precisamente nel $33 \%$ degli IDU da un minimo di 2 a un massimo di 9 tentativi e nel 9\% dei NIDU da un minimo di 2 a un massimo di 4.

La media di tentativi sul totale degli utenti osservati è di 1.5; nei pazienti IDU è di 2, nei NIDU è di 1 .

I prelievi nei pazienti IDU che hanno necessitato di più di un tentativo e sono esitati nel prelevamento dei campioni dalla vena mediana cubitale sono stati 14 su $125(11 \%)$, di cui 3 hanno richiesto una doppia sede di prelievo (Graf. 1); nei casi restanti si è proceduto con uno o più tentativi in altre sedi (Graf. 2).

Nei NIDU i prelievi in vena mediana cubitale sono riusciti con un solo tentativo ad eccezione di un caso.

4 su $53(8 \%)$ sono stati eseguiti con più di un tentativo in sedi differenti.
Graf. 1 - Distribuzione prelievi riusciti in fossa cubitale $>1$ tentativo in p.ti IDU

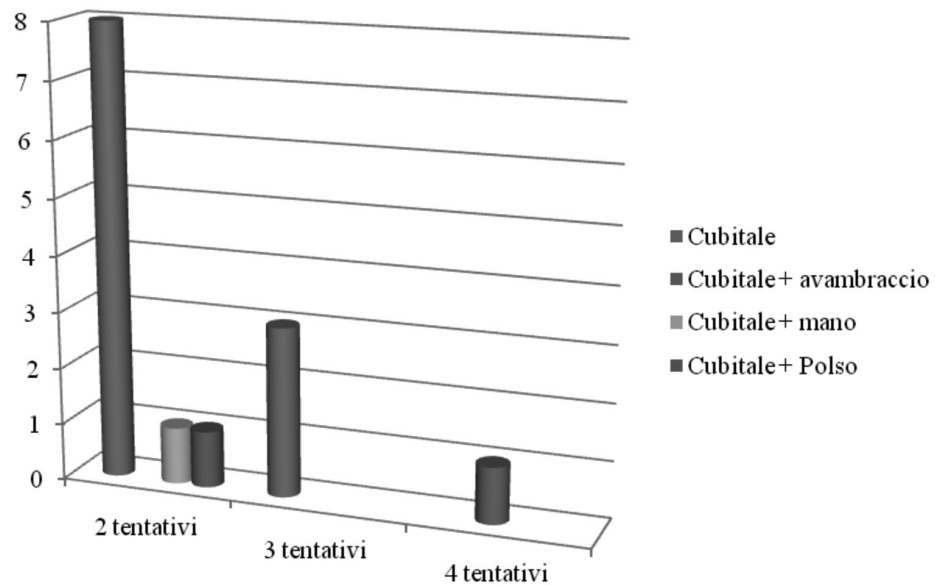

Graf. 2 - Numero di tentativi suddivisi per sede IDU

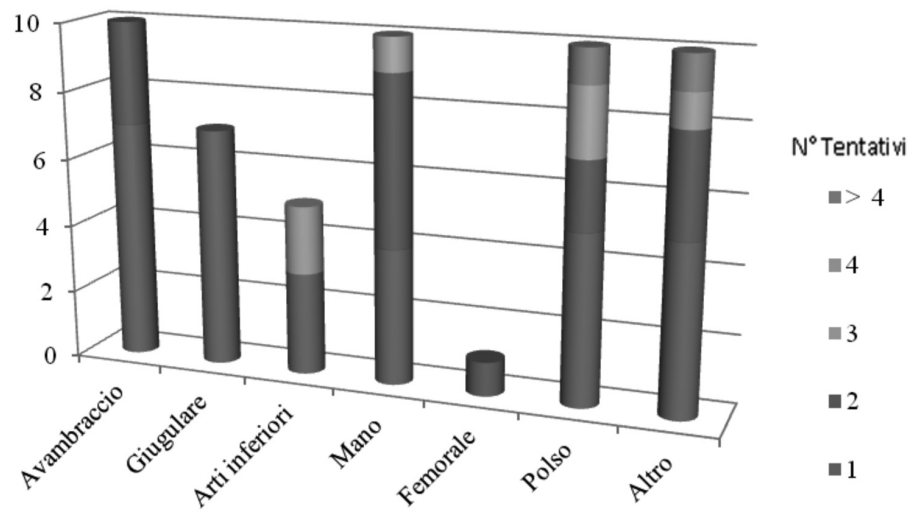

Nel $4 \%$ dei casi il prelievo è stato eseguito sugli arti inferiori. L'ecografo è stato impiegato nel $18 \%$ dei casi.

Negli IDU è stato utilizzato dopo 2 o più tentativi nel 29\% dei casi. $\mathrm{Nel} 2 \%$ dei casi si è reso necessario il prelievo arterioso in sede radiale.

La percentuale di prelievi non riusciti è del $2 \%$ e ha riguardato esclusivamente IDU

\section{Discussione}

Lo studio permette di prendere in considerazione le difficoltà che emergono nell'esecuzione dei prelievi ematici in pazienti tossicodipendenti.

Le sedute di prelievo vedono prenotati dai 10 ai 15 pazienti a seduta ma, date le difficoltà a rispettare gli appuntamenti, le difficoltà tecniche e quelle relazionali, il numero di utenti che realmente effettuano il prelievo è spesso inferiore.

Dal campione emerge una prevalenza di IDU rispetto ai NIDU poiché l'utenza che mantiene i rapporti con il Servizio è composta maggiormente da consumatori di sostanze per via iniettiva.

Gli esami più comunemente richiesti per i pazienti sono una routine di esami standard comprendente esame emocromocitometrico, quadro emocoagulativo, quadro elettroliti sierici, indici di funzionalità epatica, quadro metabolico e funzionalità renale. Per quanto riguarda I'aspetto infettivologico si effettuano esami di screening (HIV1/2 anticorpi e antigene p24, HBsAg test quan- 
titativo, HBV anticorpi anti-HBs, HBV anticorpi anti-HBc, HCV anticorpi, Treponema anticorpi totali) e talora esami di secondo livello per i pazienti con patologia già nota (HCV RNA, HIV RNA, VDRL, TPPA), pertanto il numero dei campioni da prelevare è spesso elevato.

La vena mediana cubitale è la sede preferibile di prelievo in entrambe le tipologie di pazienti, dato il grosso calibro e la minor dolorabilità (9).

Nei NIDU è di facile accesso poiché le differenti modalità di consumo di sostanze non compromettono il vaso; negli IDU è comunque la prima sede che si valuta perché non sempre è la sede privilegiata di iniezione da parte del paziente.

Molte linee guida suggeriscono un'ispezione dei siti di iniezione, come parte della valutazione clinica (5).

Da questa valutazione può emergere, nel paziente, I'uso di accessi vascolari differenti dalla mediana cubitale, ad esempio I'inoculazione della sostanza in vena femorale che, nella nostra esperienza così come in letteratura (7), è stata più volte segnalata come sito preferibile poiché di difficile visibilità. Inoltre I'uso della vena femorale è praticato non solo quando non sono più disponibili altri siti di iniezione, ma anche come sito "conveniente" per la rapida accessibilità e minore dolorabilità rispetto a vene più piccole o più scomode (12).

In altri casi invece il paziente richiede di effettuare i primi tentativi in vene differenti dalla mediana cubitale perché sede privilegiata per l'utilizzo di sostanze, o perché da lui considerata obliterata.

È in queste situazioni che prevale l'aspetto relazionale fondato sulla comunicazione efficace.

La relazione che si instaura è un veicolo per lo sviluppo di un rapporto fiduciario per il coinvolgimento del paziente nella gestione della patologia (13).

Il professionista deve quindi saper identificare i problemi percepiti dai pazienti e le relative strategie di coping, sviluppando con loro relazioni utili a migliorare la capacità di prendersi cura di sé (15). In questa ottica spetta all'infermiere valutare le singole situazioni e capire quando e se proporre la sede ritenuta più idonea, al fine di non incrinare il rapporto e rischiare di entrare in conflitto o comunque di rendere inefficace la relazione.

Da ciò si evince il motivo per cui è stato necessario in alcuni casi effettuare inizialmente il prelievo in sedi differenti dalla mediana cubitale.

In altri casi, invece, l'infermiere può trovarsi in difficoltà nel reperire accessi venosi poiché frequenti venipunture causano obliterazione e stenosi dei vasi (6) e con il tempo diventano tanto per il consumatore, quanto per il professionista, più difficili da utilizzare (3).

Si verifica pertanto in alcuni casi il fallimento dell'operazione di prelievo e si rendono necessari più tentativi in differenti sedi.

Gli arti superiori sono sempre preferibili.

Sono stati eseguiti prelievi su avambraccio, mano, polso, ma anche in sedi più inusuali quali ginocchio, gomito e zona latero-brachiale.

In alcuni casi si è reso necessario invece il prelievo in sede giugulare, femorale o il prelievo arterioso in sede radiale.

In merito agli arti inferiori, le sedi più utilizzate, seppur raramente, sono state polpaccio e piede.

Nei casi in cui il prelievo non è riuscito il paziente è stato inviato al medico di riferimento per rivalutazione ed eventuale invio a Servizi ospedalieri.

Oltre alla compromissione del patrimonio venoso, sono note altre complicanze legate all'uso endovenoso di sostanze: ascessi, celluliti, trombosi, tromboflebite settica, fistole artero-venose e pseudo aneurismi.
L'iniezione può inoltre esporre a infezioni locali o sistemiche (7-16). Non è solo la pratica iniettiva a presentare dei rischi per la salute, ma anche altre tipologie di consumo che, accompagnandosi a comportamenti a rischio, possono ad esempio incrementare il rischio di contrarre infezioni sessualmente trasmesse, pertanto la valutazione clinica dovrebbe includere domande relative agli attuali modelli di uso di sostanze e comportamenti a rischio, al fine di mettere in atto interventi di educazione sanitaria per entrambe le tipologie di pazienti.

\section{Conclusioni}

Il prelievo ematico, nella popolazione osservata, è stato effettuato prevalentemente in vena mediana cubitale.

Tuttavia si rilevano delle difficoltà su due fronti: da un lato la difficoltà tecnica di reperire negli IDU un accesso venoso idoneo (dato il patrimonio vascolare depauperato), dall'altro la difficoltà di conciliare le richieste dell'utenza con la necessità di portare a termine il prelievo senza inficiare il rapporto di fiducia professionista/paziente.

\section{Bibliografia}

1. Osservatorio europeo delle droghe e delle tossicodipendenze Relazione europea sulla droga (2015), Tendenze e sviluppi, Lussemburgo. Disponibile da: www.emcdda.europa.eu/attachements.cfm/att_213154 IT TDAT13001ITN1.pdf [consultato il 01/10/2015].

2. Karimi M., Ghaheri H., Assari S., Ahmadi K., Moghani Lankarani M., Moghani Lankarani R., Narenjiha H., Rafiey H., Tavakoli M., Jafari F. (2014), "Drug Injection to Sites other than Arm: A Study of Iranian Heroin Injectors", Front Psychiatry, 7: 5-23.

3. Alcantara A.L., Tucker R.B., McCarroll K.A. (2002), "Radiologic study of injection drug use complications", Infect Dis Clin North Am, 16(3): 713-43.

4. Darke S., Ross J., Kaye S. (2001), "Physical injecting sites among injecting drug users in Sydney, Australia", Drug Alcohol Depend, 1, 62(1): 77-82.

5. Winstock A.R., Nittis M., Whitton G., Lea T (2009), "Opioid dependent patients' experiences of and attitudes towards having their injecting sites examined", Int J Drug Policy, 20(1): 85-9.

6. Fields J.M.,Piela N.E., Au A.K., Ku B.S. (2014), "Risk factors associated with difficult venous access in adult ED patients", Am J Emerg Med, 32(10): 1179-82.

7. Coffin P.O.,Coffin L.S., Murphy S., Jenkins L.M., Golden M.R. (2012), "Prevalence and characteristics of femoral injection among Seattle-area injection drug users", J Urban Health, 89(2): 365-72.

8. Presidenza del Consiglio dei Ministri, Dipartimento Politiche Antidroga (2011), Linee di indirizzo per lo screening e la diagnosi precoce delle principali patologie infettive correlate all'uso di sostanze stupefacenti nei Dipartimenti delle Dipendenze, Roma. Disponibile da: www.iss.it/ binary/ccoa/cont/Linee_di_Indirizzo_screening.pdf [consultato il 09/10/2015].

9. Saiani L., Brugnolli A. (2011), Trattato di cure infermieristiche, IdelsonGnocchi, Napoli: 1059.

10. Maliphant J., Scott J. (2005), "Use of the femoral vein ('groin injecting') by a sample of needle exchange clients in Bristol, UK", Harm Reduct J, 15, 2(1): 6 .

11. Cavallo D., Re Luca G., Lusignani M. (2013), "Caratteristiche ed efficacia della relazione terapeutica tra infermiere e utente: una revisione della letteratura", L'infermiere, 50:6: e99-e109.

12. Nyström M. (2007), "A patient-oriented perspective in existential issues: a theoretical argument for applying Peplau's interpersonal relation model in healthcare science and practice", Scand J Caring Sci, 21(2): 282-8.

13. Salmon A.M., Dwyer R., Jauncey M., van Beek I., Topp L., Maher L. (2009), "Injecting-related injury and disease among clients of a supervised injecting facility", Drug Alcohol Depend, 101(1-2): 132-6. 